\title{
Closed Loop Control of a Gravity-assisted Underactuated Snake Robot with Application to Aircraft Wing-Box Assembly
}

\author{
Binayak Roy and H. Harry Asada \\ d'Arbeloff Laboratory for Information Systems and Technology \\ Department of Mechanical Engineering \\ Massachusetts Institute of Technology \\ Cambridge, MA 02139, USA \\ $\{$ binayak, asada\}@mit.edu
}

\begin{abstract}
Stable, nonlinear closed-loop control of a gravityassisted underactuated robot arm with $2^{\text {nd }}$ order non-holonomic constraints is presented in this paper. The joints of the hyper articulated arm have no dedicated actuators, but are activated with gravity. By tilting the base link appropriately, the gravitational torque drives the unactuated links to a desired angular position. With simple locking mechanisms, the hyper articulated arm can change its configuration using only one actuator at the base. This underactuated arm design was motivated by the need for a compact snake-like robot that can go into aircraft wings and perform assembly operations using heavy end-effecters. The dynamics of the unactuated links are essentially $2^{\text {nd }}$ order nonholonomic constraints, for which there are no general methods for designing closed loop control. We propose an algorithm for positioning the links of an $n$-link robot arm inside an aircraft wing-box. This is accomplished by sequentially applying a closed loop point-to-point control scheme to the unactuated links. We synthesize a Lyapunov function to prove the convergence of this control scheme. The Lyapunov function also provides us with lower bounds on the domain of convergence of the control law. The control algorithm is implemented on a prototype 3link system. Finally, we provide some experimental results to demonstrate the efficacy of the control scheme.
\end{abstract}

\section{INTRODUCTION}

Most assembly operations in aircraft manufacturing are currently done manually. Although aircraft are small in lot size, numerous repetitive assembly operations have to be performed on a single aircraft. The conditions are often ergonomically challenging and these result in low productivity as well as frequent injuries. Thus, there is a need to shift from manual assembly to automated robotic assembly. The following wingbox assembly illustrates this.

Fig. 1 shows a mock-up of the cross-section of an aircraft wing-box. Several assembly operations, such as burr-less drilling and fastener installations, have to be carried out inside the wing-box after the upper and lower skin panels are in place. The interior of the wing-box is accessible only through small portholes along its length. The portholes are roughly rectangular with dimensions of $45 \mathrm{~cm}$ by $23 \mathrm{~cm}$. The wingbox also has a substantial span, which varies from $1 \mathrm{~m}$ to 3 $\mathrm{m}$ depending upon the size of the aircraft. The height of the wing-box varies from about $20 \mathrm{~cm}$ to $90 \mathrm{~cm}$, depending upon the size of the aircraft. Presently, the assembly operations are

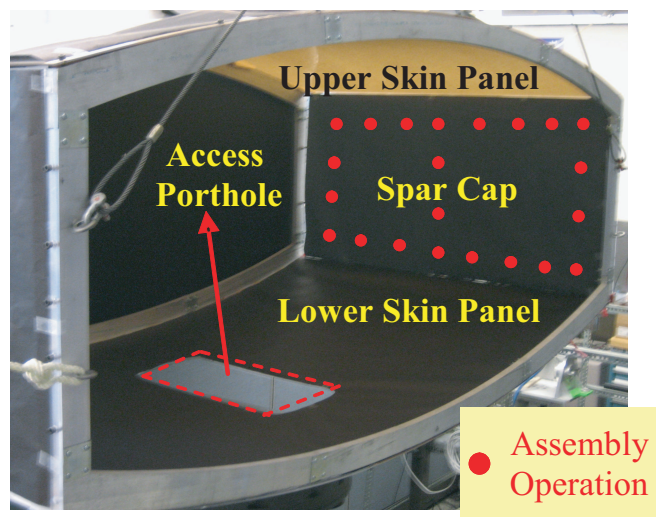

Fig. 1. Cross section of aircraft wing-box

carried out manually. A worker enters the wing-box through the small portholes and lies flat on the base, while carrying out the assembly operations. Evidently, the working conditions are ergonomically challenging.

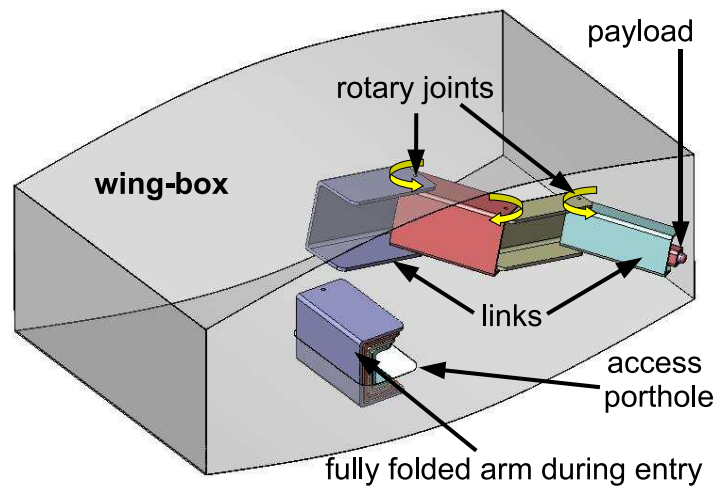

Fig. 2. Structure of robot arm

We have proposed a "Nested-Channel" serial linkage mechanism capable of operating inside an aircraft wing box [20]. The links are essentially C-channels with successively smaller base and leg lengths, as shown in Fig. 2. They are connected 
by 1 d.o.f rotary joints, the axes of which are parallel. The use of channel structures is advantageous for a number of reasons. The channels can fold into each other resulting in an extremely compact structure during entry through the porthole, as shown in Fig. 2. Once inside the wing-box, the links may be deployed to access distal points in the assembly space. The open channel structure also facilitates the attachment of a payload to the last link without increasing the overall dimensions of the arm.

The lack of a compact, powerful and high stroke actuation mechanism is the primary bottleneck in the development of the hyper articulated arm. In our previous work, we have proposed an underactuated design concept, which obviates the use of dedicated actuators for each joint. Instead, we utilize gravity for driving individual joints. This drastically reduces the size and weight of the manipulator arm. The methodology requires a single actuator for tilting the arm at the base. This single actuator can be placed outside the wing-box and can be used in conjunction with simple locking mechanisms to reconfigure the serial linkage structure.

The reconfiguration scheme is illustrated in Fig. 3, which shows a schematic of an $n$-link robot arm. The base link (link 1 ) is the only servoed link. It may be rotated about a fixed axis $Z_{0}$, which is orthogonal to the direction of gravity. All other joint axes $\left(Z_{j}, j \neq 0\right)$ are orthogonal to $Z_{0}$. They are equipped with simple on-off locking mechanisms only. The goal is to rotate link $i$ about $Z_{i-1}$ by actuating link 1 appropriately. All unactuated links except link $i$ are locked. Link 1 starts in the vertical upright position. Then it is rotated, first clockwise and then counter-clockwise, before being brought back to its vertical position. This tends to accelerate and then decelerate link $i$ due to gravity and dynamic coupling with link 1 . By controlling the tilting angle of link 1 , link $i$ can be brought to a desired position with zero velocity. Link $i$ may be locked thereafter. This procedure can be repeated sequentially for the other unactuated links. Contraction of the arm can be performed by reversing the above deployment procedure.

A considerable amount of work has been done in the area of underactuated systems [3]-[10]. Most of the work in this area deals with the planar (vertical or horizontal) case where the actuated and unactuated joint axes are parallel. In our approach, the actuated and unactuated joints are orthogonal and we can modulate the effects of gravity by controlling the actuated joint. The presence of gravity renders the nonlinear system locally controllable, as can be seen from local linearization. This ensures that we can go from any initial point to any final point in the configuration space of the unactuated coordinate. However, it is inefficient to patch together local linear control laws to traverse the entire configuration space. Moreover, any control design must ensure that the range of motion of the actuated coordinate is small, because the arm operates inside an aircraft wing-box. Earlier approaches [8]-[10] to the control of underactuated systems generate constructive global control laws applied to specific systems. Such constructive control laws cannot be directly applied to our system.

In our earlier work [21], we have proposed several motion planning algorithms suitable for the gravity-assisted under- actuated robot arm. They include parameterized trajectory planning for the actuated joint and feed-forward optimal control. These are open-loop techniques and work well in the absence of disturbances. Also, an exact knowledge of the system dynamics is needed. In particular, a good estimate of Coulomb friction is necessary for accurate position control. However, it is unrealistic to assume prior knowledge of such state dependent unknown parameters. This necessitates the development of a closed-loop control strategy for our system.

In this paper, we first explore the system dynamics to develop an understanding of the relationship between the actuated and unactuated degrees of freedom. We make important approximations to capture the dominant effects in the system dynamics so as to facilitate control design. Next, we propose a closed loop control strategy for point to point control of the unactuated coordinate. We synthesize a Lyapunov function to prove the convergence of the control law. The Lyapunov function also provides us with lower bounds on the domain of convergence of the control law. Finally, we present some experimental results which demonstrate the efficacy of the control law.

\section{SySTEM DyNAMiCS}

Fig. 3 shows a schematic of an $n$-link robot arm with one actuated (link 1) and $n-1$ unactuated links. $X_{0} Y_{0} Z_{0}$ denotes the World Coordinate Frame. The coordinate frames are attached according to the Denavit-Hartenberg convention with the $i^{t h}$ coordinate frame fixed to the $i^{t h}$ link. We seek rotation of link $i(i \geq 2)$ about the axis $Z_{i-1}$ by rotating link 1 about the horizontal axis $Z_{0}$. The angle $\theta_{1}$ denotes the tilt of link 1 relative to the fixed vertical axis $X_{0}$ and the angle $\theta_{i}$ denotes the angular position of link $i$ relative to link $i-1$.

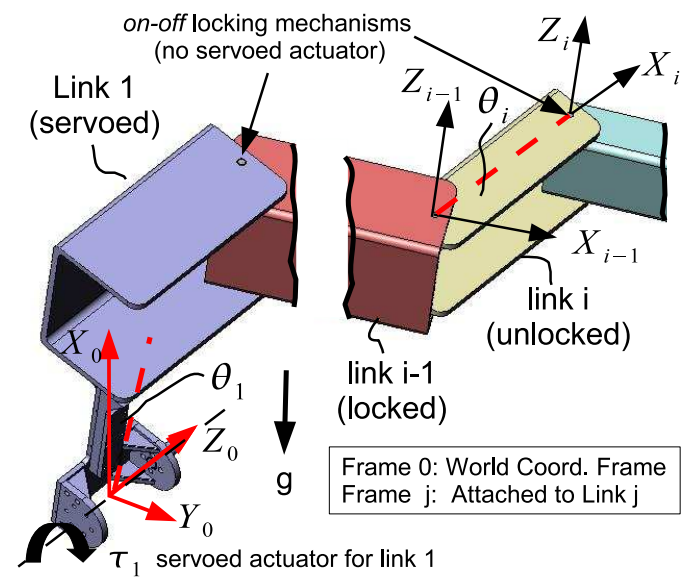

Fig. 3. Schematic of $n$-link robot arm

In the current setup, all unactuated links except link $i$ are locked. The system dynamics may be written as:

$$
\begin{array}{r}
\left.\left[\begin{array}{cc}
H_{11} & H_{i 1} \\
H_{i 1} & H_{i i}
\end{array}\right]\left[\begin{array}{c}
\ddot{\theta}_{1} \\
\ddot{\theta}_{i}
\end{array}\right]+\left[\begin{array}{c}
F_{1} \\
F_{i}
\end{array}\right]+\begin{array}{c}
{\left[\begin{array}{c}
G_{1} \\
G_{i}
\end{array}\right]=} \\
\theta_{j}=\theta_{j 0} \quad j \neq 1, i \\
\tau_{1} \\
0
\end{array}\right]
\end{array}
$$


Here $q=\left[\theta_{2}, \ldots, \theta_{n}\right]^{T},\left[H_{k l}(q)\right]$ is the $n \times n$ symmetric positive-definite inertia matrix, $\left[F_{1}\left(q, \dot{q}, \dot{\theta}_{1}\right), F_{i}\left(q, \dot{q}, \dot{\theta}_{1}\right)\right]^{T}$ represents the $2 \times 1$ vector of centrifugal and coriolis effects and $\left[G_{1}\left(q, \theta_{1}\right), G_{i}\left(q, \theta_{1}\right)\right]^{T}$ represents the $2 \times 1$ vector of gravitational effects. The torque on the actuated joint axis $Z_{0}$ is represented by $\tau_{1}$. We note that $\theta_{j 0}$ is a constant because the $j^{\text {th }}$ link $(j \neq 1, i)$ is locked. Using $F_{i}\left(q, \dot{q}, \dot{\theta}_{1}\right)=f_{i}(q) \dot{\theta}_{1}^{2}$ and $G_{i}\left(q, \theta_{1}\right)=g_{i}(q) g \sin \theta_{1}$, the second row of (1) may be written as:

$$
\ddot{\theta}_{i}=-\frac{H_{i 1}(q)}{H_{i i}(q)} \ddot{\theta}_{1}-\frac{f_{i}(q)}{H_{i i}(q)} \dot{\theta}_{1}^{2}-\frac{g_{i}(q)}{H_{i i}(q)} g \sin \theta_{1} .
$$

As shown in [3], (3) is a $2^{\text {nd }}$ order non-holonomic constraint and thus cannot be integrated to express $\theta_{i}$ as a function of $\theta_{1}$. Also, at any given time only one unactuated link (link $i$ ) is in motion. Thus, the $n$-link problem can be treated as a 2 -link problem without loss of generality. Hereafter, to simplify the algebra, we deal exclusively with the 2-link problem. For the 2-link case, we may write (3) as:

$$
\ddot{\theta}_{2}=-\frac{H_{21}\left(\theta_{2}\right)}{H_{22}\left(\theta_{2}\right)} \ddot{\theta}_{1}-\frac{f_{2}\left(\theta_{2}\right)}{H_{22}\left(\theta_{2}\right)} \dot{\theta}_{1}^{2}-\frac{g_{2}\left(\theta_{2}\right)}{H_{22}\left(\theta_{2}\right)} g \sin \theta_{1},
$$

where:

$$
\begin{aligned}
H_{12}= & M_{2}\left(z_{c 2}+d_{2}\right)\left(y_{c 2} \cos \theta_{2}+\left(x_{c 2}+a_{2}\right) \sin \theta_{2}\right) \\
& +I_{y z 2} \cos \theta_{2}+I_{x z 2} \sin \theta_{2}, \\
H_{22}= & I_{z z 2}+M_{2}\left(\left(x_{c 2}+a_{2}\right)^{2}+y_{c 2}^{2}\right), \\
f_{2}= & I_{x y 2} \cos 2 \theta_{2}+0.5\left(I_{y y 2}-I_{x x 2}\right) \sin 2 \theta_{2} \\
& +M_{2}\left(a_{1}+\left(x_{c 2}+a_{2}\right) \cos \theta_{2}-y_{c 2} \sin \theta_{2}\right) \\
& \left(\left(x_{c 2}+a_{2}\right) \sin \theta_{2}+y_{c 2} \cos \theta_{2}\right), \\
g_{2}= & -M_{2}\left(\left(x_{c 2}+a_{2}\right) \sin \theta_{2}+y_{c 2} \cos \theta_{2}\right) .
\end{aligned}
$$

$M_{2}$ denotes the mass of link 2. $I_{x y 2}$ etc. denote the moments of inertia of link 2 about a centroidal coordinate frame. The parameters $x_{c 2}, y_{c 2}, z_{c 2}$ are the coordinates of the C.O.M of link 2 in the link-attached frame. Also, $a_{2}, d_{2}$ refer to the corresponding Denavit-Hartenberg parameters.

As seen in the next section, we may choose the control torque $\tau_{1}$ in (1) so as to converge exponentially to any bounded trajectory for the actuated coordinate $\theta_{1}$. We refer to $\theta_{1}$ and its derivatives $\left(\dot{\theta}_{1}, \ddot{\theta}_{1}\right)$ in (4) as the pseudo input. The terms involving $\theta_{2}\left(H_{12} / H_{22}, f_{2} / H_{22}\right.$ and $\left.g_{2} / H_{22}\right)$ in (4) are referred to as the modulating coefficients. These modulating coefficients scale the various components of the pseudo input $\left(\theta_{1}, \dot{\theta}_{1}, \ddot{\theta}_{1}\right)$ depending on the position of the unactuated link 2 .

Fig. 4 shows the variation of the modulating coefficients in the configuration space of the unactuated coordinate. The simulation is based on parameter values taken from a 2link version of our prototype system shown in Fig. 7. The dominant term is the modulating coefficient due to gravity $\left(g_{2} / H_{22}\right)$, followed by the contribution of the inertial coupling $\left(H_{12} / H_{22}\right)$ and finally the contribution of the centrifugal coupling $\left(f_{2} / H_{22}\right)$. In view of these observations, we make the following assumptions:

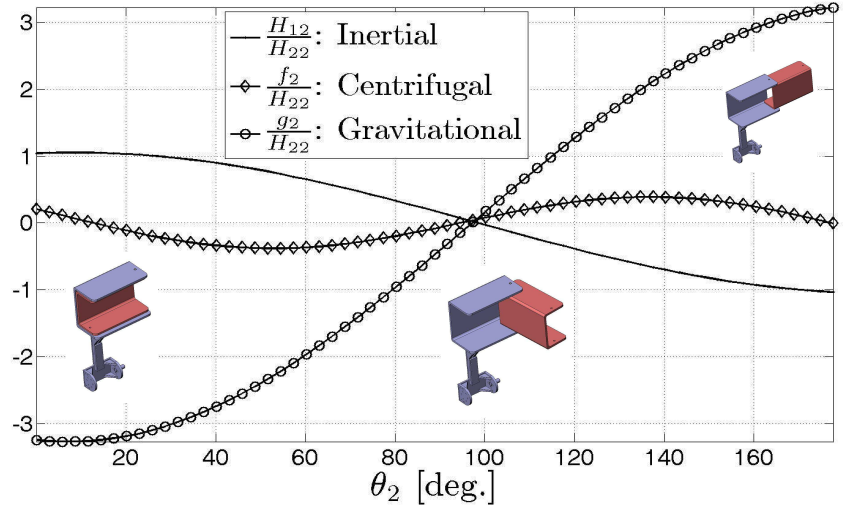

Fig. 4. Comparison of modulating coefficients over configuration space

1. Inertial coupling is neglected.

2. Centrifugal coupling is neglected.

These assumptions are valid as long as the gravitational component of acceleration $\left|g \sin \theta_{1}\right|$ is of the same (or higher) order of magnitude as compared to $\left|\ddot{\theta}_{1}\right|$ and $\left|\dot{\theta}_{1}^{2}\right|$. We validate these approximations a posteriori in the section on experimental results. Under these assumptions, the dynamics (4) may be simplified as:

$$
\ddot{\theta}_{2}=-\frac{g_{2}\left(\theta_{2}\right)}{H_{22}} g \sin \theta_{1}
$$

Using (6) and (8), we may write (9) as:

$$
\ddot{\theta}=A \sin \theta \sin \theta_{1},
$$

where:

$$
\begin{aligned}
\theta & =\theta_{2}+\alpha, \\
A & =\frac{M_{2} g \sqrt{y_{c 2}^{2}+\left(x_{c 2}+a_{2}\right)^{2}}}{I_{z z 2}+M_{2}\left(y_{c 2}^{2}+\left(x_{c 2}+a_{2}\right)^{2}\right)}, \\
\alpha & =\operatorname{atan} 2\left(y_{c 2}, x_{c 2}+a_{2}\right) .
\end{aligned}
$$

It is worthwhile to examine the physical significance of the dynamics (10). It represents a pendulum in a modulated "gravity" field. The strength of this field can be modulated as $g \sin \theta_{1}$ by controlling the angle $\theta_{1}$. The pendulum behaves as a regular or inverted pendulum depending on the sign of $\sin \theta \sin \theta_{1}$. Also, the "gravity" field may be switched off by setting $\theta_{1}=0$. This gives rise to a continuum of equilibria given by $\left[\theta=\bar{\theta}, \dot{\theta}=0, \theta_{1}=0\right]$, where $\bar{\theta}$ is arbitrary.

\section{Closed Loop Control}

\section{A. Control Law}

In this section, we propose a closed loop control law for point-to-point control of the unactuated link. The goal is to transfer the unactuated link from an initial angular position $\theta_{0}$ $\left(=\theta_{20}+\alpha\right)$ with zero initial velocity to a final angular position $\theta_{f}\left(=\theta_{2 f}+\alpha\right)$ with zero final velocity. We treat the actuated coordinate $\theta_{1}$ as a pseudo input and prescribe a feedback law in terms of the pseudo input. The formal justification of this treatment is deferred to Appendix A. 
From (10), we see that the input $\theta_{1}$ has a bounded effect on the acceleration because $\left|\sin \theta_{1}\right| \leq 1$. We propose a feedback control law of the form:

$$
\sin \theta_{1}=\frac{\sin \left(k_{1}\left(\theta_{f}-\theta\right)-k_{2} \dot{\theta}\right) \sin \theta}{k},
$$

where $k \geq 1$ and $k_{1}, k_{2}>0$ are constants. Also $\theta_{f}$ is the desired final angular position of the unactuated link. We note that $\theta_{1}$ exists because $\left|\sin \left(k_{1}\left(\theta_{f}-\theta\right)-k_{2} \dot{\theta}\right) \sin \theta / k\right| \leq 1$. Using (11) in (10) we get:

$$
\ddot{\theta}=\frac{A}{k} \sin \left(k_{1}\left(\theta_{f}-\theta\right)-k_{2} \dot{\theta}\right) \sin ^{2} \theta .
$$

The intuition behind the control law (11) is to introduce a virtual non-linear spring and damper into the system. These virtual elements introduce a stable equilibrium point $[\theta, \dot{\theta}]=$ $\left[\theta_{f}, 0\right]$ in the system dynamics. In the vicinity of the equilibrium point $\left[\theta_{f}, 0\right]$, the dynamics (12) may be approximated as:

$$
\ddot{\theta} \approx \frac{A \sin ^{2} \theta_{f}}{k}\left(k_{1}\left(\theta_{f}-\theta\right)-k_{2} \dot{\theta}\right) .
$$

The ratios $k_{1} / k$ and $k_{2} / k$ are measures of stiffness and damping respectively. Further, the multiplicative term $\sin \theta$ in (11) ensures that the sign of the acceleration $\ddot{\theta}$ in (12) is not affected by the regime of motion $(\sin \theta>0$ or $\sin \theta<0$ ). It is only affected by the deviation from the desired final state $[\theta, \dot{\theta}]=\left[\theta_{f}, 0\right]$. These intuitive notions are formalized in the proof below.

\section{B. Proof of Convergence}

Let us consider a domain $\Omega=\left\{[\theta, \dot{\theta}]:\left|k_{1}\left(\theta_{f}-\theta\right)-k_{2} \dot{\theta}\right| \leq\right.$ $\pi / 2$ and $|\theta| \leq \pi / 2\}$, and a Lyapunov function candidate (defined on $\Omega$ ):

$$
V(\theta, \dot{\theta})=\frac{B}{k_{1}} \int_{0}^{\psi} \sin x \sin ^{2}\left(\frac{x+k_{2} \dot{\theta}}{k_{1}}-\theta_{f}\right) d x+\frac{1}{2} \dot{\theta}^{2},
$$

where $\psi=k_{1}\left(\theta_{f}-\theta\right)-k_{2} \dot{\theta}, B=A / k$.

\section{Proposition:}

The control law (11) guarantees local asymptotic convergence of the state $[\theta, \dot{\theta}]$ in $(12)$ to $\left[\theta_{f}, 0\right]\left(\theta_{f} \neq 0\right)$. Further, $\exists l>0$ for which a domain of attraction of the control law is the largest connected region $\Omega_{l}=\{[\theta, \dot{\theta}]: V(\theta, \dot{\theta})<l\} \subset \Omega$.

\section{Proof:}

The scalar function $V(\theta, \dot{\theta})$ defined in (14) is positive definite in $\Omega$ because it satisfies the following conditions:

1. $V\left(\theta_{f}, 0\right)=0$.

2. $V(\theta, \dot{\theta})>0$ in $\Omega \forall[\theta, \dot{\theta}] \neq\left[\theta_{f}, 0\right]$.

The $1^{\text {st }}$ condition follows from direct substitution in (14) and noting that $[\theta, \dot{\theta}]=\left[\theta_{f}, 0\right]$ implies $\psi=0$. The $2^{\text {nd }}$ condition follows by noting that $\sin x>0$ for $\pi / 2 \geq x>0$ and $\sin x<$ 0 for $-\pi / 2 \leq x<0$. Thus, for $0<|\psi| \leq \pi / 2$ :

$$
\int_{0}^{\psi} \sin x \sin ^{2}\left(\frac{x+k_{2} \dot{\theta}}{k_{1}}-\theta_{f}\right) d x>0 .
$$

Henceforth, we abbreviate $V(\theta, \dot{\theta})$ as $V$. It is convenient to rewrite (14) as:

$$
\begin{aligned}
V= & \frac{B}{2}\left[\frac{k_{1}\left(\cos \psi \cos 2 \theta-\cos 2\left(\frac{\psi}{k_{1}}+\theta\right)\right)-2 \sin \psi \sin 2 \theta}{k_{1}^{2}-4}\right] \\
& +\frac{B}{2 k_{1}}(1-\cos \psi)+\frac{1}{2} \dot{\theta}^{2}, \quad k_{1} \neq 2 .
\end{aligned}
$$

The time derivative of (15) is given by:

$$
\begin{aligned}
\dot{V}= & \frac{\partial V}{\partial \theta} \dot{\theta}+\frac{\partial V}{\partial \dot{\theta}} \ddot{\theta} \\
= & -\frac{B^{2} k_{2} \sin ^{2} \theta}{k_{1}\left(4-k_{1}^{2}\right)}\left[\left(2-k_{1}^{2} \sin ^{2} \theta\right) \sin ^{2} \psi\right. \\
& \left.+k_{1} \sin \psi\left(\sin 2 \theta \cos \psi-\sin 2\left(\frac{\psi}{k_{1}}+\theta\right)\right)\right]
\end{aligned}
$$

It may be shown that $\dot{V} \leq 0$ in $\Omega$ for all $k_{1}, k_{2}>0$. In the interest of brevity, we just prove this assertion for $k_{1}=1$ and $k_{2}>0$. We further show that $\exists l_{0}>0$, such that $\Omega_{l_{0}} \subset \Omega$. Substituting $k_{1}=1$ in (16) and after some rearrangement we get:

$$
\begin{aligned}
\dot{V}= & -\frac{B^{2} k_{2}}{3} \sin ^{2} \theta(1-\cos \psi)\left[3 \sin ^{2} \theta \cos \psi(1-\cos \psi)\right. \\
& +(2 \sin \theta \cos \psi+\sin \psi \cos \theta)^{2}+(\sin \psi \cos \theta+\sin \theta)^{2} \\
& \left.+\sin ^{2} \theta \cos ^{2} \psi\right]
\end{aligned}
$$

We note the $0 \leq \cos \psi \leq 1$ in $\Omega$. Thus, the expression in square brackets in (17) is always non-negative. Hence, $\dot{V} \leq 0$ in $\Omega$. Also, from (17):

$$
\begin{aligned}
\dot{V} & =0 \\
\Rightarrow \theta & =0 \text { or } \psi=0
\end{aligned}
$$

Using (18) in (12) we get:

$$
\dot{V}=0 \Rightarrow \ddot{\theta}=0
$$

From (18) and (19), the largest invariant set where $\dot{V}=0$ is given by $\left\{[\theta, \dot{\theta}]=[0,0] \cup\left[\theta_{f}, 0\right]\right\}$. Using La Salle's invariant set theorem, we conclude that the state $[\theta, \dot{\theta}]$ converges to $[\theta=$ $0, \dot{\theta}=0]$ or $\left[\theta=\theta_{f}, \dot{\theta}=0\right]$.

The choice of $l_{0}$ is illustrated graphically in Fig. 5. We used (14) for the simulation with the parameters $k_{1}=1, k_{2}=1$, $B=32$ and $\theta_{f}=30^{\circ}$. For these parameters, we obtain $l_{0}=$ 0.54 and $\Omega_{l_{0}}$ is the largest connected region within $\Omega$ such that $V(\theta, \dot{\theta})<l_{0}$. Once again, it follows from La Salle's invariant set theorem that $\Omega_{l_{0}}$ is a domain of attraction for the largest invariant set.

It remains to establish the stability of the equilibrium points. We show that $[\theta=0, \dot{\theta}=0]$ is unstable and $\left[\theta=\theta_{f}, \dot{\theta}=0\right]$ is a stable equilibrium point for $k_{1}=k_{2}=1$. We note that there are other choices of $k_{1}, k_{2}$ for which these conclusions hold and the current choice only serves to simplify the algebra. From (15):

$$
\frac{\partial^{2} V}{\partial \theta^{2}}=0 \text { and } \frac{\partial^{3} V}{\partial \theta^{3}} \neq 0 \text { at }[\theta=0, \dot{\theta}=0] .
$$

This implies that $[\theta=0, \dot{\theta}=0]$ is not a local minimum 


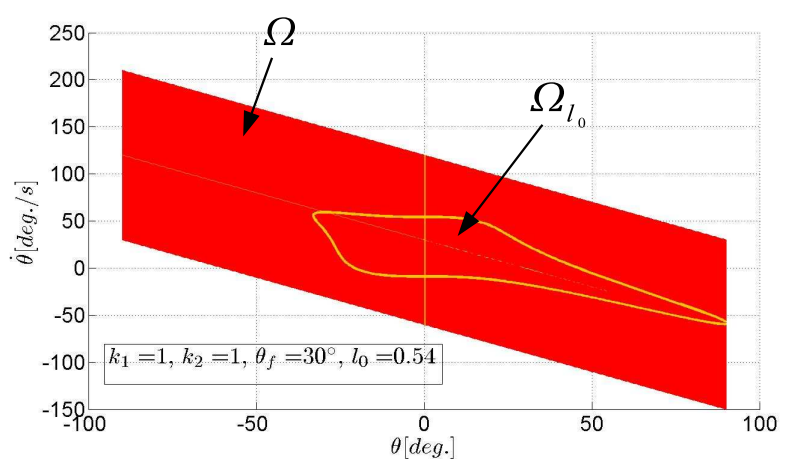

Fig. 5. Domain of Convergence

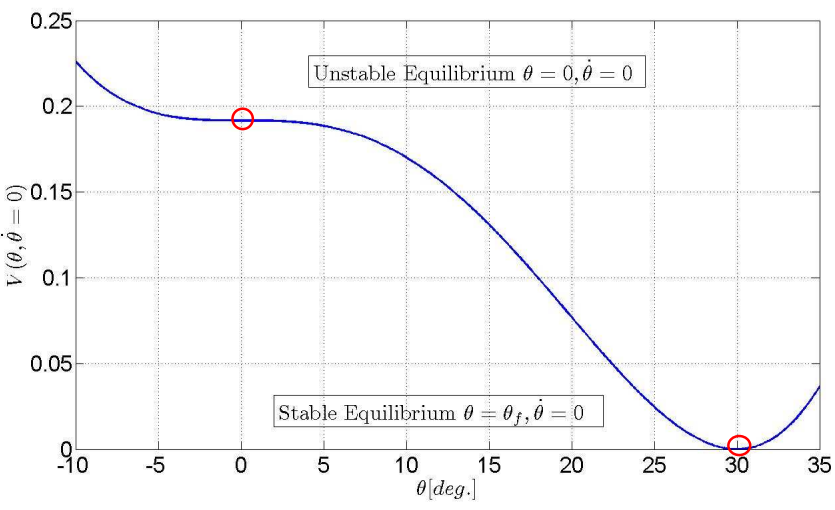

Fig. 6. Stable and unstable equilibria of system dynamics

for $V$ and thus an unstable equilibrium point. We note that this conclusion does not follow from linearization because the linearized system has zero eigenvalues at $[\theta=0, \dot{\theta}=0]$. Once again, from (15):

$$
\nabla^{2} V=\left[\begin{array}{cc}
B \sin ^{2} \theta_{f} & B \sin ^{2} \theta_{f} \\
B \sin ^{2} \theta_{f} & B \sin ^{2} \theta_{f}+1
\end{array}\right] \text { at }\left[\theta=\theta_{f}, \dot{\theta}=0\right] .
$$

This implies that $\nabla^{2} V$ is positive definite and $\left[\theta=\theta_{f}, \dot{\theta}=0\right]$ is a local minimum for $V$ and thus a stable equilibrium point. These ideas are illustrated in Fig. 6 for the case $k_{1}=1$, $k_{2}=1, B=32$ and $\theta_{f}=30^{\circ}$. Thus the state $[\theta, \dot{\theta}]$ in (12) converges to $\left[\theta_{f}, 0\right]$ as long as it does not start from $[0,0]$.

\section{IMPLEMENTATION AND EXPERIMENTS}

We conducted position control experiments on a prototype system with 3 links which is shown in Fig. 7. The link mechanism, which operates inside the wing-box, is shown in Fig. 7a. The links are essentially C-channels which are serially connected by 1 d.o.f rotary joints. Link 1 is the only servoed link. Links 2 and 3 are equipped with on-off pneumatic brakes. The relative angular position of the links are measured using optical encoders placed at the rotary joints. They have a resolution of 1000 ppr.

The actuation mechanisms for link 1 operate completely outside the wing-box and are shown in Fig. 7b. They comprise a servoed tilting mechanism and a servoed azimuthal positioning mechanism. The tilting mechanism is used to tilt link 1 relative to a vertical axis. Depending on the state (on or off) of the pneumatic brakes, the unactuated links (2 and 3) may be deployed by exploiting gravity and dynamic coupling with link 1. The azimuthal positioning mechanism is used for angular positioning of the entire link mechanism inside the wing-box and serves to expand the workspace of the robot arm. This mechanism is used after the links have been deployed using the tilting mechanism. The pneumatic brakes are in the on state when the azimuthal positioning mechanism is in use. Both mechanisms have harmonic drive gearing (100:1) coupled to AC servomotors $(0.64 \mathrm{Nm}, 3000 \mathrm{rpm})$. In the experiments that follow, the azimuthal positioning mechanism is not used. We only use the tilting mechanism to deploy the links and verify the proposed control law.

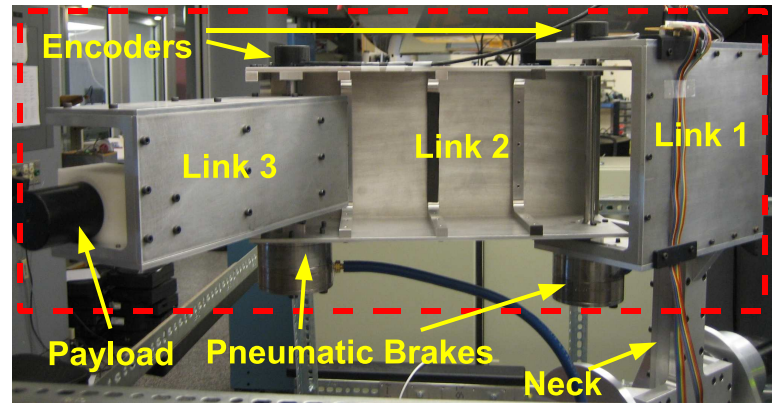

(a) Link mechanism (operates inside wing-box)

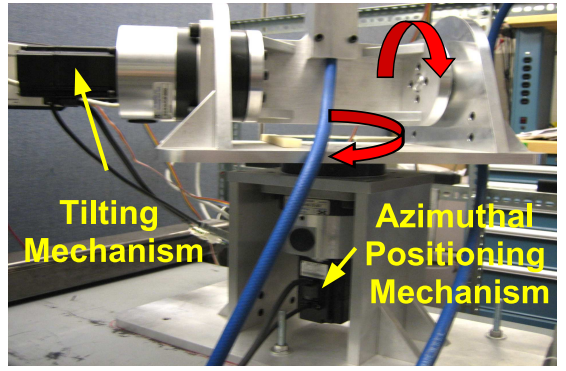

(b) Actuation Mechanisms (operate outside wing-box)

Fig. 7. 3-link prototype arm

The dynamical system (10) corresponding to our experimental setup has the parameters $A=32.8 s^{-2}$ and $\alpha=-3.2^{\circ}$. The experimental results are illustrated in Fig. 8. The goal was to move link 2 from an initial position $\theta_{20}=35^{\circ}$ to a desired final position of $\theta_{2 f}=50^{\circ}$. Link 3 was kept fixed at $30^{\circ}$ relative to link 2 . The controller parameter values in (11) were set at $k=12, k_{1}=1.2$ and $k_{2}=0.2 s$. It may be verified that these parameters ensure that the initial position lies within the domain of convergence. The scaling factor of $k=12$ was used to restrict the amplitude of $\theta_{1}$ to less than $1.5^{\circ}$. A small amplitude of $\theta_{1}$ is very important in practice because the arm operates inside an aircraft wing. There are other choices of $k_{1}$ and $k_{2}$ which ensures convergence of the control law. For example, a higher value of $k_{2}$ would imply less overshoot and slower convergence.

The actual final position of the arm was $\theta_{2}=50.5^{\circ}$ as shown in Fig. 8a. The tilt trajectory of link 1 is shown in 
Fig. 8b. The maximum tilt is $1.3^{\circ}$ which is small enough for operation inside the wing-box. Fig. 8c shows a comparison of the gravitational, inertial and centrifugal contributions on the angular acceleration of link 2 . The gravitational contribution clearly dominates the other effects. This demonstrates, $a$ posteriori, the validity of the approximations made in our dynamic modeling.

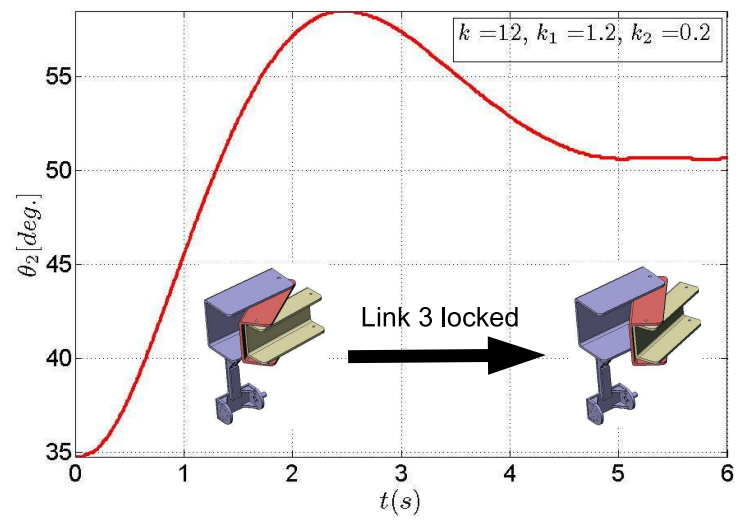

(a) Link 2 trajectory (unactuated joint)

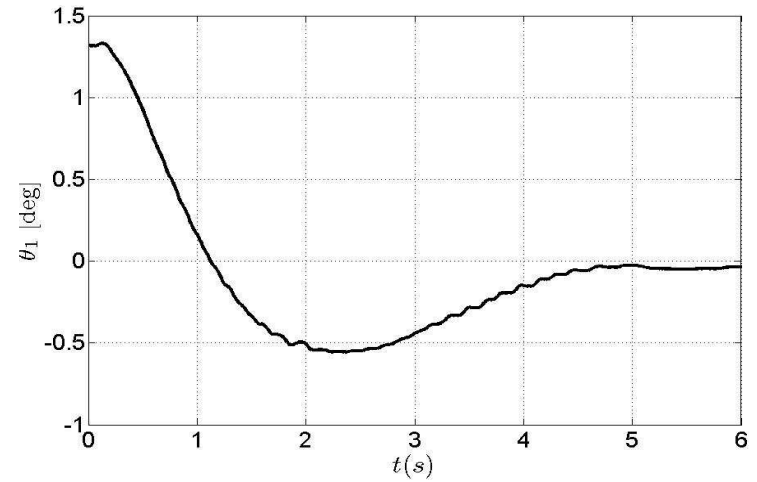

(b) Link 1 trajectory (servoed joint)

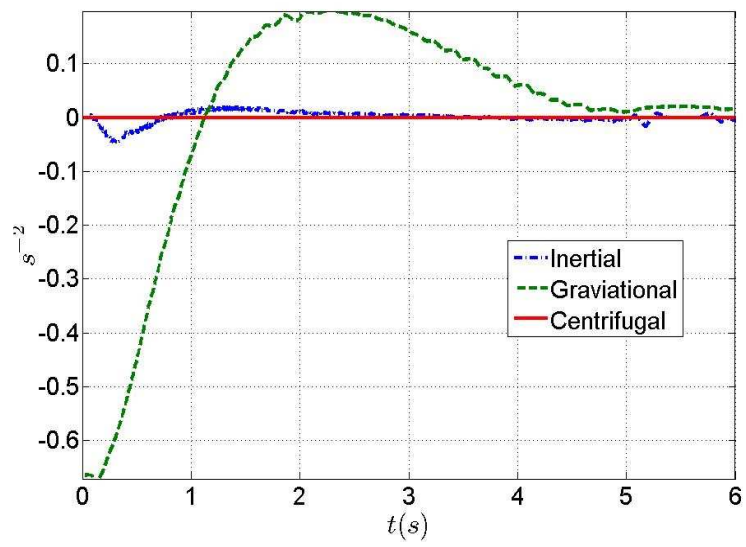

(c) Comparison of gravitational, inertial and centrifugal effects

Fig. 8. Position control experiment on 3-link prototype

The control law (11) demonstrates reasonable positioning accuracy of the unactuated links. The performance is achieved without any knowledge of Coulomb friction or the dynamics introduced by the flexible hose supplying air to the pneumatic brakes. This is a significant improvement compared to the open loop motion planning schemes explored in our earlier work. Such schemes required frequent tuning of friction coefficients and other parameters related to the dynamics of the hose.

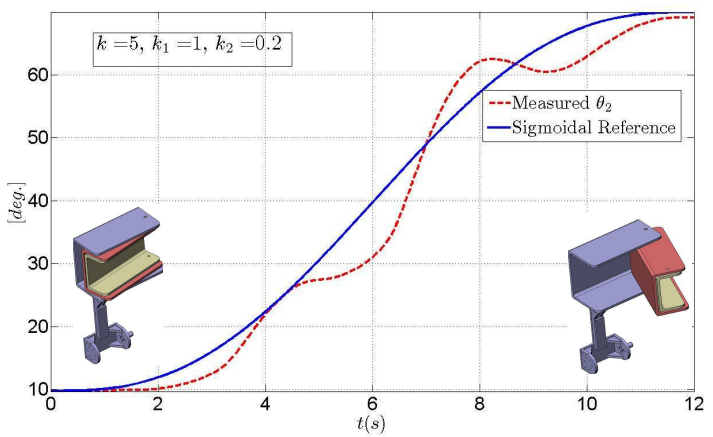

(a) Link 2 trajectory (unactuated joint)

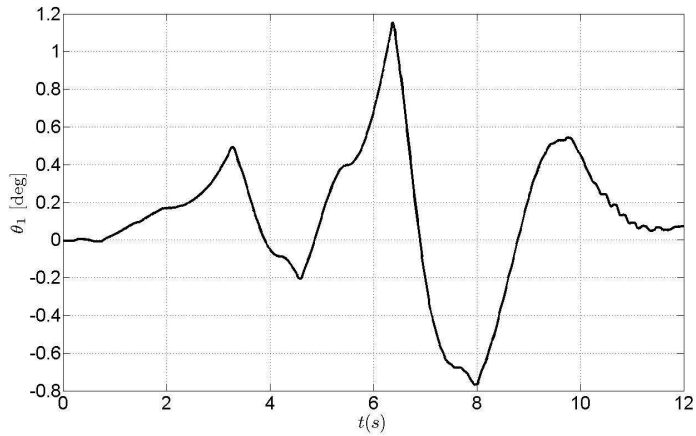

(b) Link 1 trajectory (servoed joint)

Fig. 9. Experimental results for modified control law using sigmoidal reference trajectory

A primary drawback of the proposed control law arises from the conflicting requirements of small amplitude of tilt of link 1 and small steady state error for link 2 . This is readily seen from (11). If link 2 starts at $\theta_{0}$ with zero initial velocity, the initial tilt of link 1 is given by:

$$
\sin \theta_{10}=\frac{\sin \left(k_{1}\left(\theta_{f}-\theta_{0}\right)\right) \sin \theta}{k}
$$

$\theta_{10}$ may be large if the amplitude of motion $\left|\theta_{f}-\theta_{0}\right|$ is large. To achieve smaller values of $\theta_{10}$, the scaling factor $k$ may be increased or the gain $k_{1}$ may be reduced. As noted before, the ratio $k_{1} / k$ is a measure of the stiffness of the virtual non-liner spring introduced by the controller. Increasing $k$ and reducing $k_{1}$ would result in lower stiffness. This would lower the speed of convergence and also increase the steady state error induced by Coulomb friction.

We address this issue by replacing the fixed reference $\theta_{f}$ in (11) by a time varying reference $\theta_{r e f}(t)$ starting at $\theta_{0}$ and changing smoothly to $\theta_{f}$. In particular, the reference may be a sigmoidal trajectory given by:

$$
\theta_{\text {ref }}(t)=\left\{\begin{array}{c}
\theta_{0}+\left(10 \mu^{3}-15 \mu^{4}+6 \mu^{5}\right)\left(\theta_{f}-\theta_{0}\right) \\
\mu=\frac{t}{t_{f 1}}, \quad 0 \leq t \leq t_{f 1} \\
\theta_{f} \quad t \geq t_{f 1}
\end{array}\right.
$$


We may choose $t_{f 1}$ to set a desired average speed of motion $\left|\theta_{f}-\theta_{0}\right| / t_{f 1}$. Substituting (21) in (11), we obtain the modified control law:

$$
\sin \theta_{1}=\frac{\sin \left(k_{1}\left(\theta_{\text {ref }}(t)-\theta\right)-k_{2} \dot{\theta}\right) \sin \theta}{k} .
$$

We applied the control law (22) to our prototype system. The goal was to move link 2 from an initial position $\theta_{20}=$ $10^{\circ}$ to a desired final position of $\theta_{2 f}=70^{\circ}$. Link 3 was kept fixed at $0^{\circ}$ relative to link 2 . The controller parameter values in (22) were set at $k=5, k_{1}=1$ and $k_{2}=0.2 \mathrm{~s}$, $t_{f 1}=12 s$. The experimental results are shown in Fig. 9. The actual final position was $69.7^{\circ}$ at the end of $12 \mathrm{~s}$, as shown in Fig. 9a. The tilt trajectory of link 1 is shown in Fig. 9b. The maximum amplitude of tilt of link 1 was $1.1^{\circ}$ which is within the acceptable limits.

\section{CONClusion}

We have addressed the problem of closed loop point-topoint control of a gravity assisted underactuated robot arm. The arm is particularly well suited to high payload assembly operations inside an aircraft wing-box. We proposed a closed loop control algorithm for point-to-point control of the unactuated links. A Lyapunov function was synthesized to prove the convergence of the control law. The Lyapunov function also provides us with lower bounds on the domain of convergence of the control law.

The control algorithm was applied to a prototype 3-link robot arm. The experimental results showed reasonable performance of the control law in the absence of prior knowledge of friction and other unmodelled dynamical effects. We further proposed a modified control law to handle the conflicting requirements of small tilt of the actuated link and low steady state error of the unactuated links. The efficacy of the modified control law was demonstrated on the prototype system.

The modified control law results in a non-autonomous dynamical system. Our current proof has to be suitably modified to prove asymptotic convergence of the output using the modified control scheme. Also, a lower steady state error in the position of the unactuated links is desirable. These issues will be addressed in future work.

\section{ACKNOWLEDGMENTS}

The authors gratefully acknowledge the support provided by the Boeing Company. The first author would like to thank Dr. Jun Ueda for many helpful discussions.

\section{APPENDIX A}

We justify the treatment of the actuated coordinate $\theta_{1}$ as a pseudo input. We denote the desired trajectory of $\theta_{1}$ by $\theta_{1 d}$. From (11), $\theta_{1 d}=\sin ^{-1}\left(\sin \left(k_{1}\left(\theta_{f}-\theta\right)-k_{2} \dot{\theta}\right) \sin ^{2} \theta / k\right)$. The dynamics of the actuated coordinate $\theta_{1}$ may always be feedback linearized by choosing the control torque as:

$$
\tau_{1}=\frac{\ddot{\theta}_{1 d}-2 \lambda \dot{\tilde{\theta}}_{1}-\lambda^{2} \tilde{\theta}_{1}}{N_{11}}+F_{1}+G_{1}+\frac{N_{12}}{N_{11}}\left(F_{2}+G_{2}\right),
$$

where:

$$
\begin{gathered}
{\left[\begin{array}{ll}
N_{11} & N_{12} \\
N_{12} & N_{22}
\end{array}\right]=\left[\begin{array}{cc}
H_{11} & H_{i 1} \\
H_{i 1} & H_{i i}
\end{array}\right]^{-1},} \\
\tilde{\theta}_{1}=\theta_{1}-\theta_{1 d} \text { and } \lambda>0 .
\end{gathered}
$$

Using (23) in (1), the error dynamics of the actuated coordinate is given by:

$$
\ddot{\tilde{\theta}}_{1}+2 \lambda \dot{\tilde{\theta}}_{1}+\lambda^{2} \tilde{\theta}_{1}=0
$$

Let us define $x=[\theta, \dot{\theta}]^{T}$ and $y=\left[\tilde{\theta}_{1}, \dot{\tilde{\theta}}_{1}\right]^{T}$. The dynamics of the unactuated coordinate $(x)$ and the error dynamics of the actuated coordinate $(y)$ may be written in cascade form as $\dot{x}=f(x, y)$ and $\dot{y}=g(y)$. Here, $f(x, y)=$ $\left[\dot{\theta}, A \sin \theta \sin \left(\theta_{1 d}+\tilde{\theta}_{1}\right)\right]^{T}$ and $g(y)=\left[\dot{\tilde{\theta}}_{1},-2 \lambda \dot{\tilde{\theta}}_{1}-\lambda^{2} \tilde{\theta}_{1}\right]^{T}$. We note that $f(x, y)$ is globally Lipschitz and the linear subsystem $\dot{y}=g(y)$ is globally exponentially stable. Also, we have proved that the non-linear subsystem $\dot{x}=f(x, 0)$ is asymptotically stable using La Salle's Theorem. It follows from Sontag's Theorem [22], [24] that the cascade system is locally asymptotically stable for an appropriate choice of $\lambda$.

\section{REFERENCES}

[1] S. Hirose and M. Mori, "Biologically Inspired Snake-like Robots Robotics and Biomimetics," in Proc. of ROBIO, Aug. 2004, pp 1-7.

[2] A. Wolf, H. B. Brown, R. Casciola, A. Costa, M. Schwerin, E. Shamas and H. Choset, "A mobile hyper redundant mechanism for search and rescue tasks," Proc. of IROS, Oct. 2003, vol. 3, pp 2889-2895.

[3] G. Oriolo and Y. Nakamura, "Free-joint manipulators: motion control under second-order nonholonomic constraints," in Proceedings of IROS, Nov. 1991, vol. 3 pp 1248-1253.

[4] J. Hauser and R. M. Murray, "Nonlinear controller for nonintegrable systems: the acrobot example," in Proc. American Contr. Conf., 1990, pp 669-671.

[5] M. W. Spong, "Partial feedback linearization of underactuated mechanical systems," in Proc. of IROS, Sep. 1994 v 1, pp 314-321.

[6] M. W. Spong, "Swing up control of the acrobot," in Proc. of ICRA, Mar. 1994, v 3, pp 2356-2361.

[7] K. M. Lynch and M. T. Mason, "Dynamic underactuated nonprehensile manipulation," in Proc of IROS, Nov. 1996 v 2, pp 889-896.

[8] H. Arai, K. Tanie and N. Shiroma, "Nonholonomic control of a threedof planar manipulator," in IEEE Trans. Robot. Automat., vol. 14, no. 5, pp 681-695, Oct. 1998.

[9] H. Arai, "Position control of a 3-dof manipulator with a passive joint under a Nonholonomic constraint," in Proc. of IROS, Nov. 1996 v 1, pp $74-80$.

[10] K. M. Lynch, N. Shiroma, H. Arai and K. Tanie, "Collision-free trajectory planning for a 3-dof robot with a passive joint," in Int. J. Robot. Res. vol. 19, no. 12, Dec. 2000, pp. 1171-1184.

[11] R. W. Brockett, "Asymptotic stability and feedback stabilization," in Differential Geometric Control Theory, R. W. Brockett, R. S. Millman, and H. J. Sussmann, Eds. Boston, MA: Birkhäuser, 1983, pp. 181-191.

[12] T. Suzuki, M. Koinuma, and Y. Nakamura, "Chaos and nonlinear control of a nonholonomic free-joint manipulator," in Proc. of IROS, 1996, pp. $2668-2675$.

[13] T. Suzuki and Y. Nakamura, "Nonlinear control of a nonholonomic free joint manipulator with the averaging method," in Proc. 35th IEEE Int. Conf. Decision Contr., 1996, pp. 1694-1699.

[14] A. De Luca and G. Oriolo, "Motion planning under gravity for underactuated three-link robots, in Proc. of IROS, 2000, pp.139-144.

[15] A. Jain and G. Rodriguez, "An analysis of the kinematics and dynamics of underactuated manipulators," in IEEE Trans. Robot. Automat., vol. 9, pp. 411-422, Aug. 1993

[16] R. Mukherjee and D. Chen, "Control of free-flying underactuated space manipulators to equilibrium manifolds," in IEEE Trans. Robot. Automat., vol. 9, pp. 561-570, Oct. 1993. 
[17] Y. Nakamura and R. Mukherjee, "Nonholonomic path planning of space robots via a bidirectional approach," in IEEE Trans. Robot. Automat., vol. 7, pp. 500-514, Aug. 1991.

[18] E. Papadopoulos and S. Dubowsky, "Failure recovery control for space robotic systems," in Proc. American Contr. Conf., 1991, pp. 1485-1490.

[19] E. Papadopoulous, Path planning for space manipulators exhibiting nonholonomic behavior, in Proc. IEEE/RSJ Int.Workshop Intell. Robots Syst. (IROS92), 1992, pp. 669-675.

[20] B. Roy and H. Asada, "An underactuated robot with a hyper-articulated deployable arm working inside an aircraft wing-box," in Proc. of IROS, August 2005, pp 4046-4050.

[21] B. Roy and H. Asada, "Dynamics and control of a gravity-assisted underactuated robot arm for assembly operations inside an aircraft wingbox," in Proc. of ICRA, May 2006, pp 701-706.

[22] E. D. Sontag, "Remarks on stabilization and input-to-state stability," in Proc. 28th IEEE Conf. Decision Contr., vol. 2, pp. 1376-1378, 13-15 Dec. 1989.

[23] H. J. Sussmann, "A general theorem on local controllability," in SIAM J. Contr. Optimization, vol. 25, no. 1, pp. 158-194, 1987.

[24] H. J. Sussmann and P.V. Kokotovic, "The peaking phenomenon and the global stabilization of nonlinear systems," in IEEE Trans. Automat. Contr., vol. 36, no. 4, pp. 424-440, April 1991.

[25] I. Kolmanovsky and N. H. McClamroch, "Developments in nonholonomic control problems," in IEEE Contr. Syst., vol. 15, pp. 20-36, 1995.

[26] R. M. Murray, Z. Li and S. S. Sastry, A Mathematical Introduction to Robotic Manipulation, CRC Press. 